\title{
A retrograde double-labeling technique for light microscopy A combination of axonal transport of cholera toxin B-subunit and a gold-lectin conjugate
}

\author{
T.J.H. Ruigrok *, T.M. Teune, J. van der Burg, H. Sabel-Goedknegt \\ Department of Anatomy, Erasmus University, P.O. Box 1738, 3000 DR Rotterdam, The Netherlands
}

Received 5 September 1994; revised 23 February 1995; accepted 24 February 1995

\begin{abstract}
A light microscopical, non-fluorescent, retrograde double-labeling technique is described. Cholera toxin B-subunit (CTb) and a conjugate of wheatgerm agglutinin and bovine serum albumin coupled to $10 \mathrm{~nm}$ gold particles (gold-lectin) are both excellent retrograde tracers and, when visualized by means of immunohistochemistry and silver intensification, respectively, may be readily identified within the same cell.

This light microscopical retrograde double-labeling technique is illustrated in rat with experiments designed to investigate the collateralisation (1) of vestibular neurons to the spinal cord and oculomotor complex, (2) of spinal neurons to the left and right lateral reticular nucleus, and (3) of inferior olivary neurons to the uvula of the cerebellum.

Advantages over fluorescent double-labeling experiments are found in the fact that the diaminobenzidine reaction product as well as the silver/gold deposits do not fade and can be examined in counterstained sections. Moreover, the injection sites can be kept quite small and may be guided by electrophysiological recording through the injection pipette.
\end{abstract}

Keywords: Retrograde tracing; Fluorescent tracer; Axon collateral; Vcstibular nuclei; Lateral reticular nucleus; Cerebellum; Inferior olive

\section{Introduction}

The introduction of the fluorescent retrograde double-labeling technique by Kuypers and collaborators (Bentivoglio et al., 1980a,b; Huisman et al., 1983; Keizer et al., 1983; Kuypers et al., 1977, 1980) has aided neuroanatomical research enormously since it became possible to study the collateralisation of axons. More recently, the number of fluorescent tracers to choose from has increased dramatically and made triple or multiple retrograde labeling possible. Combinations of fluorescent tracers with wheatgerm agglutinatedhorseradish peroxidase (WGA-HRP) have been employed also (Wigston and Kennedy, 1987; Kitao et al., 1989). However, there are a number of disadvantages in employing fluorescent tracers. The analysis of the

\footnotetext{
" Corresponding author: Tel.: (31) 10-408-7296; Fax: (31) 10-4365780.
}

material is usually arduous since it has to be performed with the aid of fluorescence microscopy in which cytoarchitectural boundaries are difficult to identify. When tracers are used with different excitation wavelengths, filters may have to be changed. Moreover, fluorescence is prone to fading, especially after prolonged storage or illumination. In addition, fluorescent tracers are known to be rather toxic to both the animal (they may result in considerable tissue necrosis) (see e.g.: Keizer et al., 1983) and the environment, and are, with some exceptions, difficult to dissolve. Because of this last property, injections with these fluorescent tracers (such as Fast Blue, Diamino Yellow and Nuclear Yellow), frequently result in large injection sites. Finally, it is usually not possible to record neuronal activity through the injection pipette to help establishing the optimal injection site.

For these reasons we searched for a combination of two retrogradely transported tracers that: (1) are both easily detected with the light microscope; (2) are read- 
ily differentiated from one another even when present in the same neuron; (3) can be combined with counterstaining; (4) can be deposited in small-sized injection sites; (5) allow recording of neuronal activity through the injection pipettes.

We report here that the combination of cholera toxin B-subunit ( $\mathrm{CTb}$ ) and a goldsol coupled to the lectin WGA that was conjugated to bovine serum albumine (BSA) fulfills these demands. The use of CTb as a retrograde tracer was introduced by Luppi and et al. (1990) and gold-lectin conjugates have first been used by Menétrey (1985).

\section{Methods}

\subsection{Preparation and injection of tracers}

\subsubsection{Cholera toxin B-subunit}

Retrograde tracing with $\mathrm{CTb}$ results in the visualisation of the soma and a large part of the dendritic tree of the labeled neurons (Luppi et al., 1990; Shinonaga et al., 1992). In addition, CTb is known to be transported in an anterograde fashion. We used iontophoretic injections of CTb as originally published by Luppi et al. (1990). However, since we predominantly made use of the low-salt variety of $\mathrm{CTb}$ (List Biological Lab Product \#104, lot \#CVXG-15A), there was no need to desalt and exchange buffers. Thus, $0.5 \mathrm{mg}$ of lyophilized $\mathrm{CTb}$ was dissolved in $50 \mu \mathrm{l}$ of distilled water to a final concentration of $1 \% \mathrm{CTb}$ in $0.2 \mathrm{M}$ sodium phosphate ( $\mathrm{pH} 7.5$ ), which were aliquotted in 4 $\mu l$ quantities and stored in the freezer.

Glass micropipettes containing an inner filament (outer diameter: $2 \mathrm{~mm}$; Clark Electromedical Instruments) with tips broken at $12-15 \mu \mathrm{m}$ were backfilled with the aliquotted $\mathrm{CTb}$ solution using a Hamilton syringe. The micropipette was connected to a $1.0 \mathrm{ml}$ syringe with a Luer Lock adapter for catheders (Unimed, Geneva, Switzerland) and pressure was applied manually to the back of the micropipette to the point were CTb solution oozed out of the pipette tip. In this way, air bubbles trapped in the shank of the pipette were removed. A silver wire, insulated except for the tip, connected the solution with a Grass preamplifier. Electrophysiological recordings were conventionally monitored and stored on tape (Fig. 1). After determination of the injection location, the pipette was connected to an iontophoresis device, capable of delivering constant positive current pulses ( $7 \mathrm{~s}$ on, $7 \mathrm{~s}$ off) of 4 $\mu \mathrm{A}$ for a period of $15-30 \mathrm{~min}$. At the end of this period, the pipettes were left in situ for $5-10 \mathrm{~min}$ to

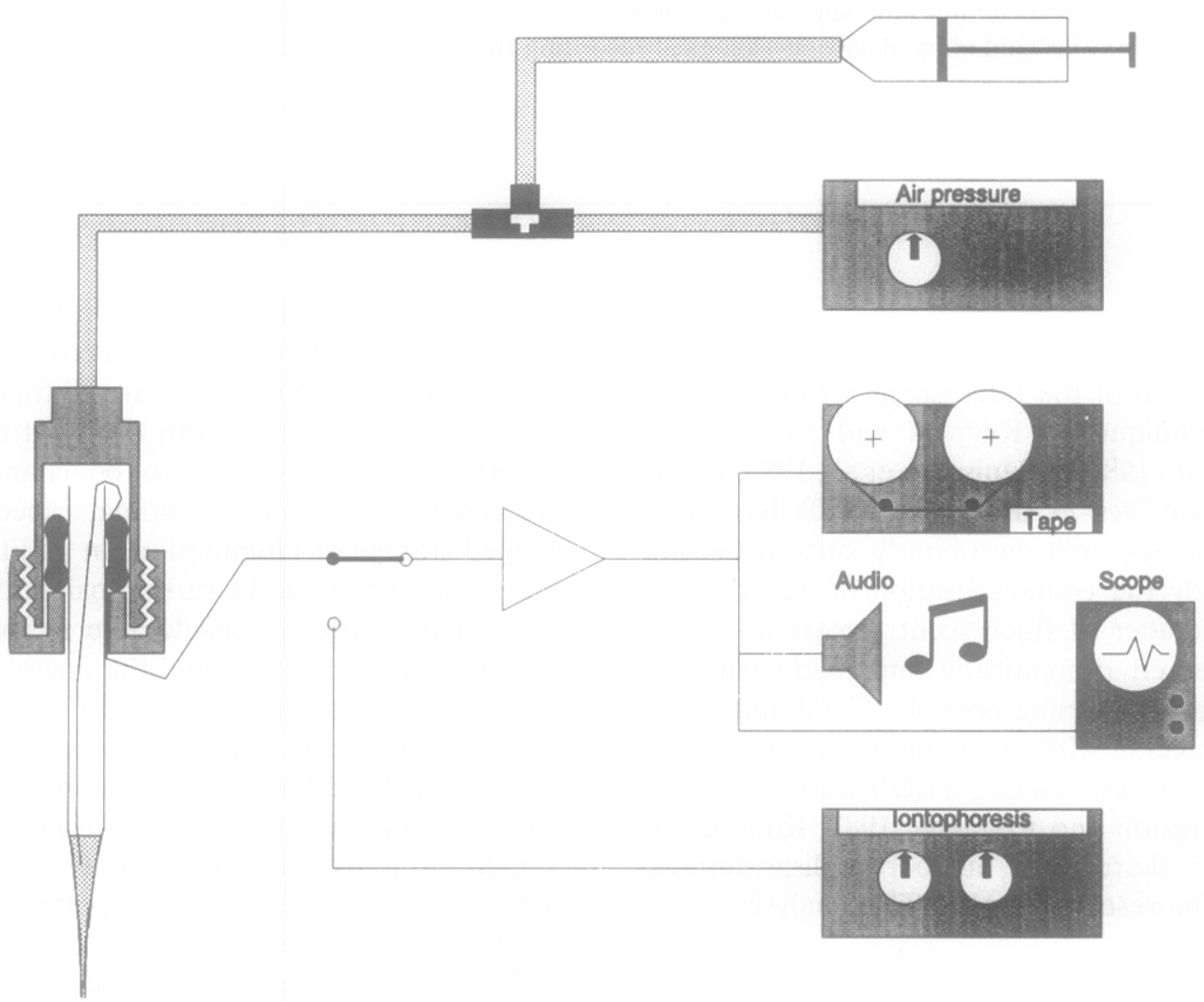

Fig. 1. Schematic diagram of the experimental set-up. Note that electrical signals recorded at the pipette tip can be monitored after which ejection of the tracer may take place by either iontophoresis or pressure injection. 
minimize leakage of the tracer along the pipette track. In some types of experiments $\mathrm{CTb}$ was pressure-injected (e.g., in the spinal cord).

\subsubsection{Goldsol coupled to WGA-BSA conjugate}

Gold-lectin conjugates have been introduced as a neuroanatomical tracer by Menétrey and colleagues (Basbaum and Menétrey, 1985; Menétrey and I ee, 1985; Menétrey, 1987) and have been demonstrated to be transported in a strictly retrograde direction (Llewellyn-Smith et al., 1992). Initially, goldsol was coupled to WGA-HRP or WGA-apoHRP. Here, we report that $10 \mathrm{~nm}$ goldsol (Aurion) coupled to a conjugate of WGA and BSA also acts as an excellent, but much cheaper, retrograde tracer. The following steps (modified after Roth, 1983) were performed to prepare a tracer of a good, reproducible, quality.

Step 1: preparation of the WGA-BSA conjugate. Dissolve $1 \mathrm{mg} \mathrm{WGA} \mathrm{(Sigma)} \mathrm{and} 4 \mathrm{mg} \mathrm{BSA}$ in $0.25 \mathrm{ml}$ of $0.005 \mathrm{M} \mathrm{NaCl}$. Adjust $\mathrm{pH}$ to 7.0 with either $0.2 \mathrm{M}$ $\mathrm{H}_{3} \mathrm{PO}_{4}$ or $0.2 \mathrm{M} \mathrm{K}_{2} \mathrm{CO}_{3}$. Add $50 \mu \mathrm{l}$ of $0.25 \%$ glutaraldehyde (E.M. grade) and stir for $2 \mathrm{~h}$ at room temperature. Finally, add $12.2 \mathrm{ml}$ of $0.005 \mathrm{M} \mathrm{NaCl}$ and dialyze overnight in $0.005 \mathrm{M} \mathrm{NaCl}$. Total volume of the protein solution is $12.5 \mathrm{ml}$ and contains $5 \mathrm{mg}$ of protein.

Step 2: preparation of the WGA-BSA-gold conjugate. Prepare the following solutions in clear glass or plastic vials:

$90 \mu$ l of protein $+10 \mu$ l of $0.005 \mathrm{M} \mathrm{NaCl}$;

$70 \mu \mathrm{l}$ of protein $+30 \mu \mathrm{l}$ of $0.005 \mathrm{M} \mathrm{NaCl}$;

$50 \mu l$ of protein $+50 \mu$ l of $0.005 \mathrm{M} \mathrm{NaCl}$;

$30 \mu$ l of protein $+70 \mu$ l of $0.005 \mathrm{M} \mathrm{NaCl}$;

$10 \mu l$ of protein $+90 \mu l$ of $0.005 \mathrm{M} \mathrm{NaCl}$.

Adjust $10 \mathrm{~nm}$ colloidal gold solution (Aurion) to $\mathrm{pH}$ 9.9 (with $0.2 \mathrm{M} \mathrm{K}_{2} \mathrm{CO}_{3}$ ) and add $1 \mathrm{ml}$ of goldsol to each vial. Vortex and wait for $2 \mathrm{~min}$. Determine which vial remains just clear and use this combination of protein content and goldsol solution to prepare the definite WGA-BSA-gold conjugate. In this way, we usually found that $3.75 \mathrm{ml}$ of the protein conjugate should be added to $85 \mathrm{ml}$ of the $\mathrm{pH}$-adjusted goldsol. Other protein/goldsol volume combinations also yielded qualitatively good tracers. Stir for $2 \mathrm{~min}$ and add $1 \%$ Carbowax (PEG 20M) to a final concentration of $0.05 \%$.

Centrifuge at $45000 \mathrm{~g}$ for 30 minutes. Aspirate and discard supernatant, leaving approximately $10 \%$ of the initial volume. Add Carbowax $0.05 \%$ to original volume, resuspend loose part of sediment and centrifuge again at $45,000 \times g$ for $30 \mathrm{~min}$. Carefully aspirate the supernatant, leaving in total approximately $50-100 \mu$ l of the WGA-BSA-gold conjugate, which can be used for injection. The conjugate can be stored for several months at $4^{\circ} \mathrm{C}$ without losing its retrograde transport capabilities.

Gold-lectin conjugates are not suited to be delivered iontophoretically. Therefore, in order to be able to make restricted injections a pressure device was used (Gibson et al., 1987). A small amount (about $1 \mu \mathrm{l}$ ) of the WGA-BSA-gold solution was sucked into a glass micropipette (tip diameter: $12-15 \mu \mathrm{m}$ ) by applying vacuum to the back of the pipette, which was Luer-locked to the pressure delivering device (Fig. 1). We empirically established that electrophysiological recordings through the WGA-BSA-gold solution could be made possible by introducing a thin (diameter: 75 $\mu \mathrm{m})$ and except for the very tip, insulated silver wire into the pipette prior to filling (Fig. 1). After establishing the optimal injection location, pressure pulses of varying intensity were applied to inject the tracer. The total injected volume (in $\mathrm{nl}$ ) was estimated to be equal to $1 / 4 \pi d^{2} h$, where $d$ is the approximate diameter (in $\mathrm{mm}$ ) of the gold-lectin meniscus in the shank of the injection pipette and $h$ the displacement (in $\mathrm{mm}$ ) of the meniscus during injection. In this way, volumes ranging between 10 and $1000 \mathrm{nl} \mathrm{WGA-BSA-gold} \mathrm{could}$ be reliably injected.

\subsection{Surgical procedure}

Injections were made in male Wistar rats $(200-250$ g) which had been anesthetized with pentobarbital ( 120 $\mathrm{mg} / \mathrm{kg}$, i.p.) and were mounted in a stereotactic device. Injection sites were roughly determined with the aid of the stereotactic atlas of Paxinos and Watson (1986) but were verified by recording neuronal activity typical of region to be injected through the injection pipette. Target areas were combinations of inferior olivary nucleus, lateral reticular nucleus, cerebellar cortex, oculomotor area and cervical spinal cord. These areas were reached by drilling a hole in the parietal bone (oculomotor area) or by enlargement of the foramen magnum dorsalwards (after retraction of neck muscles from the squamosal part of the occipital bone) and opening of the dura mater and reflecting it laterally. The spinal cord was reached by performing a laminectomy at $\mathrm{C} 4$ or $\mathrm{C} 5$.

After both injections were made, the wounds were sutured, and the rats were allowed to recover from surgery. All rats were able to eat and drink at the first postoperative day and could move freely within their cages. Nevertheless, they were checked daily in order to be able to provide pain medications if behavior would indicate so. Depending on the length of the studied pathways, the rats survived for 3-8 days.

\subsection{Perfusion}

Prior to perfusion, the rats were given a lethal dose of sodium pentobarbital $(200 \mathrm{mg} / \mathrm{kg})$. The brain was perfusion-fixed by cannulation of the ascending aorta 
A

CTb

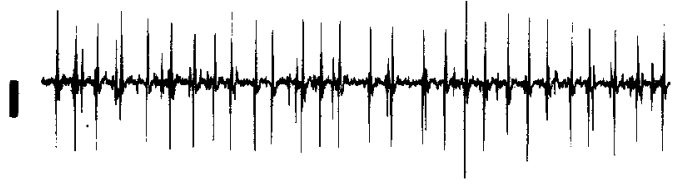

B

WGA-BSA-Gold

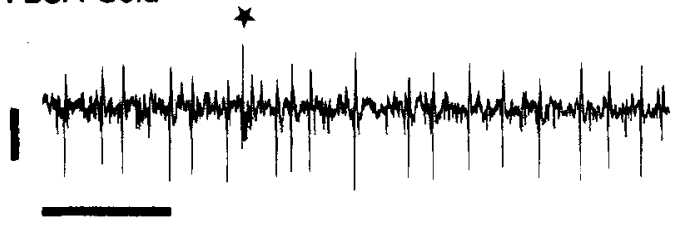

C

$\mathrm{CTb}$

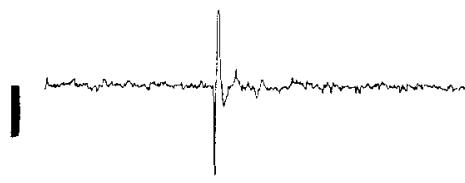

D

WGA-BSA-Gold

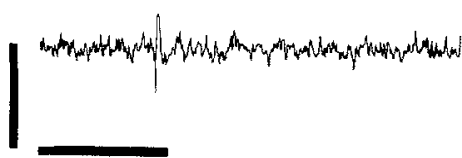

Fig. 2. Examples of electrophysiological recordings obtained with CTb-filled (A and C) and gold-lectin-filled (B and D) pipettes in the cerebellar cortex ( $A$ and $B$ ) and inferior olive ( $C$ and D). Note the most likely single-unit Purkinje cell recording in $B$ displaying numerous simple spikes as well as a complex spike (star). C and D show the characteristic extracellular action potential of olivary neurons consisting of a negative-positive going spike followed by several spikelets. Horizontal bar equals $100 \mathrm{~ms}$ in $\mathrm{A}$ and $\mathrm{B}$ and $20 \mathrm{~ms}$ in $\mathrm{C}$ and $\mathrm{D}$. Vertical bar equals $0.1 \mathrm{mV}$.

and by subsequent infusion of $200 \mathrm{ml}$ of $0.05 \mathrm{M}$ phosphate buffer ( $\mathrm{pH} 7.4$ ) containing $0.8 \% \mathrm{NaCl}, 0.8 \%$ sucrose and $0.4 \%$ glucose followed by $1000 \mathrm{ml}$ of fixative made up of $4 \%$ freshly prepared paraformaldehyde and $0.1 \%$ glutaraldehyde in $0.05 \%$ phosphate buffer containing $4 \%$ sucrose. After perfusion the brain and spinal cord were extracted and postfixed in the same fixative for 3-5 h. They were rinsed and stored overnight in $10 \%$ sucrose in phosphate buffer, embedded in gelatin (10\%) which was hardened for $3 \mathrm{~h}$ in formaldehyde ( $4 \%$ and containing $30 \%$ sucrose) and again stored overnight in $30 \%$ sucrose in phosphate buffer.

\subsection{Histology}

Sections were serially cut at $40 \mu \mathrm{m}$ on a freezing microtome and collected in glass vials containing 0.05 M phosphate buffer (pH 7.4). Due to the high density of the black gold particles, the location and size of the WGA-BSA-gold injection site could be easily evaluated during cutting. Cases with clearly misplaced WGA-BSA-gold injections could be discarded allready at this stage. Free-floating sections were incubated in anti-CTb (1:15,000; List Laboratories) in Tris buffer containing $0.5 \mathrm{M} \mathrm{NaCl}$ and $0.5 \%$ Triton $X-100$
(TBS,$+ \mathrm{pH} 8.6$ ) for 3 nights and days (at $4^{\circ} \mathrm{C}$ ). The sections were subsequently incubated in biotinylated donkey anti-goat (B-DAG, List Laboratories; 1:2000 in TBS + ) for $2 \mathrm{~h}$, rinsed and reacted with the avidinebiotine complex (ABC Elite kit, Vector) for $2 \mathrm{~h}$ and, finally, reacted with diaminobenzidine (DAB: $37.5 \mathrm{mg}$ in $150 \mathrm{ml}$ of Tris- $\mathrm{HCl}, \mathrm{pH} 7.6$, with $25 \mu 130 \% \mathrm{H}_{2} \mathrm{O}_{2}$ ) for $30 \mathrm{~min}$. Enhancement of the WGA-BSA-gold label was achieved with silver intensification (Aurion) which was performed on floating sections after the $\mathrm{CTb}$ immunohistochemistry. Sections were subsequently mounted serially on chrome-gelatinized glass slides, air dried, counterstained with thionin, cleared in xylene and coverslipped with Permount.

\section{Results}

\subsection{Recording of neuronal activity through the injection pipette}

One of the advantages of the currently used tracers is the possibility to make small injections. However, when doing so, the exact location of the injection is very important. Therefore, relying on stereotactic coordinates may result in wrong placements. Hence,

\footnotetext{
Fig. 3. Color microphotograps showing the CTb pressure injection into the left half of the cervical spinal cord (A) and the WGA-BSA-gold injection into the oculomotor area (B). Note that the injection is centered around the left trochlear nucleus. $C$ shows a photomontage of the contralateral vestibular nuclei. Note the abundance of gold-labeled neurons in the medial vestibular nucleus (adjacent to the fourth ventricle: IV) as well as CTb-labeled neurons within the large-celled part of the medial vestibular nucleus. Some of these neurons also contain gold/silver particles (arrows). Note that, on this side of the brain, the lateral vestibular nucleus (dorsal to the magnocellular part of the medibl vestibular nucleus) contains no labeled neurons. $D$ and $E$ show higher magnifications of single- and double-labeled neurons depicted in $C$. Bar equals 1 mm in $A$ ( $B$ same magnification), $100 \mu \mathrm{m}$ in $C$, and $25 \mu \mathrm{m}$ in $\mathrm{D}$ ( $E$ same magnification).
} 

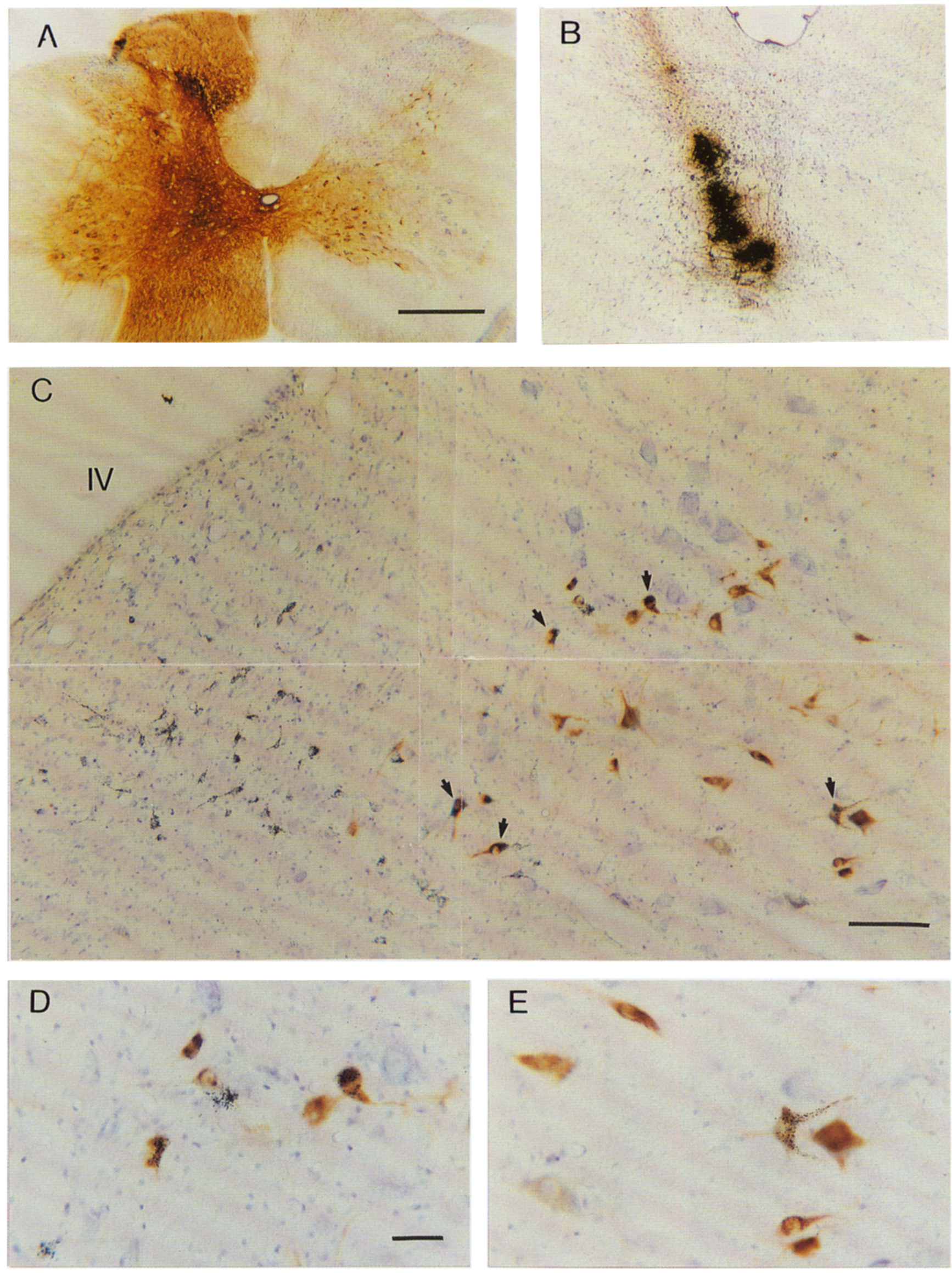

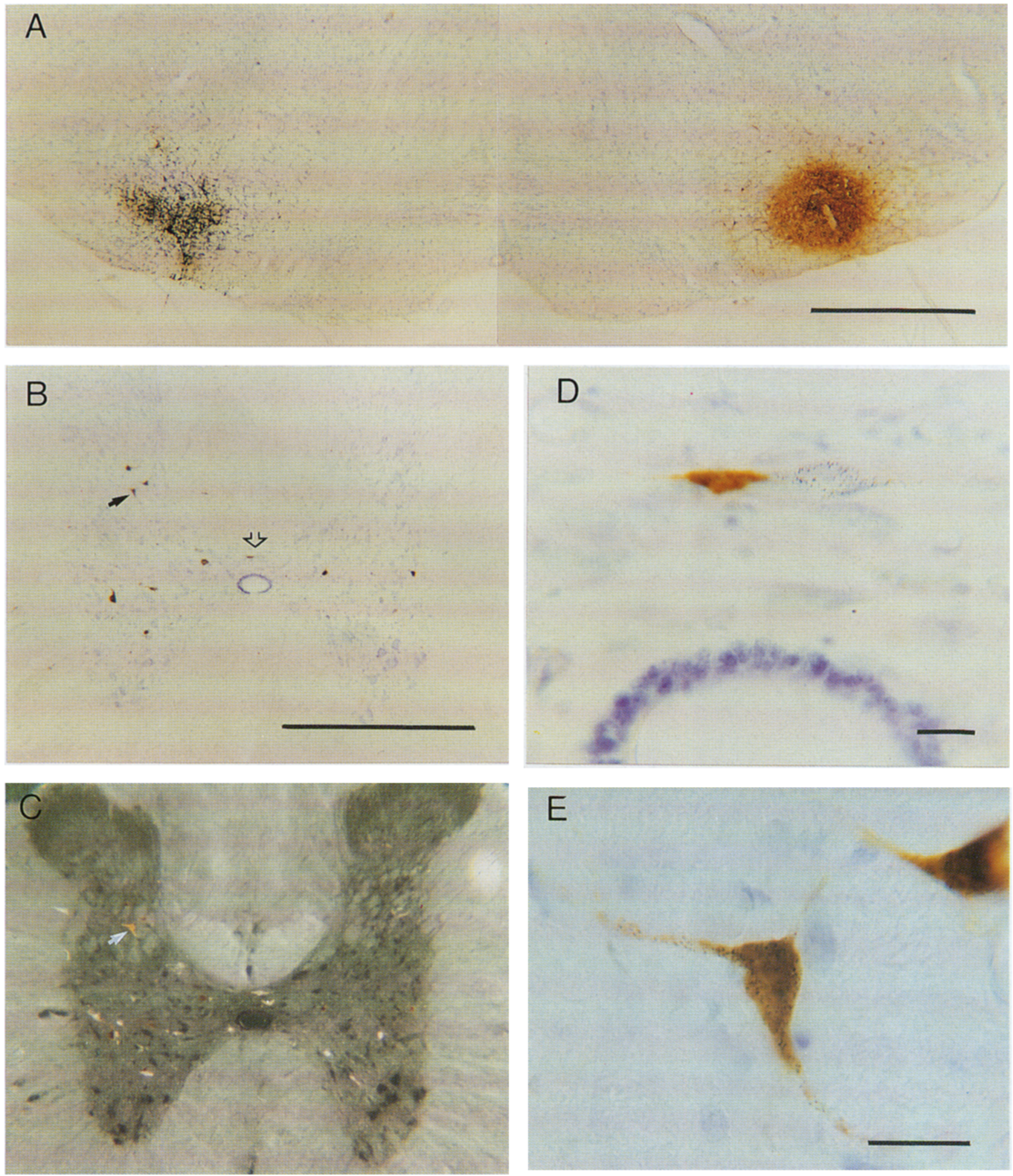
recording of neuronal activity through the injection pipette may aid in determining the exact location of tracer injection. Multi-unit, but also single-unit, recordings may be obtained with CTh-filled as well as with WGA-BSA-gold-filled pipettes as is shown for recordings made within the cerebellar cortex and inferior olive (Fig. 2). Although the signal-to-noise ratio is better for the $\mathrm{CTb}$ pipette, the slow and irregular spontaneous firing of olivary action potentials (Ito, 1984) can also be registered and appreciated with the WGA-BSA-gold pipette. Using these recordings it was possible to make selective injections with one tracer in the rostral part of the medial accessory olive and place the other one in caudal part of the same olivary subdivision or to place one tracer in the dorsal accessory and the other one in the medial accessory olive at the same rostrocaudal level without making more than 1 or 2 injection tracks for each tracer. With the objective of studying the collateralization of olivocerebellar fibers it turned out to be useful to rely on recordings of complex spike activity, typical for Purkinje cells, in order to be able to deliver both tracers, directly adjacent to one another, in the uvula of the cerebellar cortex without the risk of injecting only the white matter or granule cell layer (Fig. 5).

\subsection{Injection sites}

\subsubsection{Cholera toxin B-subunit}

$\mathrm{CTb}$ was routinely delivered iontophoretically. We used the low-salt CTb (List) although initially a number of iontophoretic injections were made with normal CTb that was desalted according to Luppi et al. (1990). In both cases the injection site appeared similar and conformed to the description given by Luppi et al. (Figs. 3A and 5A). From the small and circumscript injection site numerous axons could be traced either distally into their terminals or proximally unto their somata, indicating that CTb is transported in anterograde as well as retrograde direction. However, and in contrast to the observations by Luppi et al. (1990), we are not convinced that passing, undamaged fibers do not take up and transport $\mathrm{CTb}$, e.g., when $\mathrm{CTb}$ was injected into the inferior olive, especially after longer survival times, labeled neurons could be seen within the contralateral inferior olive as well as labeled climbing fibers within the ipsilateral cerebellar cortex. How- ever, the staining intensity was always much lower compared to either the climbing fiber labeling in the contralateral cerebellar cortex or of retrograde labeling of neurons known to project to the inferior olive. Reducing the survival time to 3 or 4 days (when tracing connections within the brain stem) appears to minimize the problem of labeling passing fibers. Notwithstanding, this potential handicap, which may also occur with many other tracers, should be considered when designing a particular experiment.

In some experiments, CTb was pressure-injected. Injection of a total of $100-200 \mathrm{nl}$, divided over 2 or 3 tracks into either the right or left side of the spinal cord results in an injection site that covered most of the injected side but did not spread to the other half of the cord (Fig. 4A).

\subsection{2. $W G A-B S A-$ gold}

All sections analysed for gold-lectin tracer were routinely silver-intensified. Therefore, in the following account, gold-labeling actually refers to silver-intensified gold unless noted otherwise. As reported elsewhere (Basbaum and Menétrey, 1987; Llewellyn-Smith et al., 1992) gold-labeled tracers result in small, welldefined injection sites (Figs. 3A and 4B), especially when compared to similar amounts of HRP-based tracers or CTb. However, we have noted that WGABSA-gold possesses an enhanced affinity for fiber bundles, e.g., it was frequently seen that large quantities of the gold-lectin tracer were predominantly located within the fiber bundles between the neuropil lamellae of the inferior olive. Nevertheless, undamaged, passing fibers do not appear to take up this tracer as was also concluded by Basbaum and Menétrey (1987).

\subsection{Retrograde double-labeling}

Retrograde labeling with $\mathrm{CTb}$ results in light- or dark brown-stained neuronal cell bodies from which frequently dendrites can be identified for considerable distances. Therefore, the neuropil surrounding the labeled somata may contain numerous thin labeled profiles which, depending upon the particular injection and analysis site, may result from retrograde labeling of distal dendrites, anterograde labeling of afferents, or anterograde labeling of recurrent axon collaterals from

\footnotetext{
Fig. 4. A: color microphotomontage showing the WGA-BSA-gold pressure and iontophoretic CTb injection site in the left and right LRN, respectively. Note that the injections are approximately the same size and are centered on similar parts of the LRN. B shows a color microphotograph of a section through the thoracic part of the spinal cord. Note several CTb-labeled neurons located ipsi- as well as contralaterally of the CTb injection site. At this magnification, gold-labeled neurons cannot be detected. C shows the same section with darkfield illumination. Note that now the gold/silver-labeled neurons can be easily spotted. D demonstrates that at higher magnification both CTb as well gold-labeled neurons may be readily detected as shown for these labeled neurons located directly dorsal to the central canal (and indicated in $B$ by an open arrow). E shows a double-labeled neuron indicated by an arrow in B and C. Bar equals $1 \mathrm{~mm}$ in $A$ and $B$ (C same magnification as B), and $25 \mu \mathrm{m}$ in $\mathrm{D}$ and $\mathrm{E}$.
} 
retrogradely labeled neurons. However, in all cases it is possible to identify the retrogradely labeled neuronal somata without difficulty (e.g., Figs. 3 and 4).

WGA-BSA-gold injections, contrary to the $\mathrm{CTb}$ injections, only result in retrogradely labeled neurons. Axonal labeling was never observed from the injection site, either in anterograde or retrograde direction. The retrogradely labeled cells were easily identified, although the degree of dendritic labeling was somewhat less compared to the $\mathrm{CTb}$ labeling (Figs. $3 \mathrm{E}$ and $4 \mathrm{E}$ ). Darkfield illumination can be employed to search for labeling with low-power objectives (Fig. 3C).

Since both the colour of the labeling as well as the labeling pattern are very different from one another, it is remarkably easy to determine whether a particular neuron contains both tracers (Figs. 3D,E and 4C,D,E). Only in some very small or very intensely CTb-stained neurons it may be difficult to identify small amounts of gold/silver particles. In these cases it is possible to rely on darkfield illumination in order to be able to determine whether a particular neuron is double-labeled.

In order to illustrate the possiblities, advantages and possible pitfalls of this double-labeling technique 3 types of experiments will be described; however, they will not be discussed in depth, since complete papers dealing with these specific topics are currently being prepared.

\subsubsection{Labeling in the vestibular nuclei after injections in the spinal cord and oculomotor area}

The vestibular nuclei contain the elements that link the sensory neurons of the vestibular ganglion to the motoneurons of the eye muscles as well as to the motoneurons of the spinal cord, in particular of the neck musculature. Many neurons in the vestibular nuclei can be related to eye as well as to head movements; however, anatomical data on collateralization are sparse. The present experiment was designed to provide data on collateralization of vestibular neurons in the rat. $\mathrm{CTb}$ was pressure-injected into the left half of the cervical spinal cord at $\mathrm{C} 4$ or $\mathrm{C} 5$. Three injection tracks were made and a total of $150 \mathrm{nl}$ of $\mathrm{CTb}$ was injected, but the injection site did not appear to extend

Fig. 5. Semidiagramatic representation of the distribution of retrogradely labeled neurons in the inferior olivary complex after small CTb (laterally) and WGA-BSA-gold (medially) injections in lobule IXb (uvula) of the contralateral side. Note that most gold-labeled neurons are located in the caudomedial part of the $\beta$-subnucleus and within the lateral part of the caudal part of the medial accessory olive (MAO). CTb neurons are located within the rostrolateral part of the $\beta$-subnucleus, the dorsomedial cell column (DMCC) and in the dorsomedial group (DM) of the principal olive (PO). Only 2 double-labeled neurons were encountered which were located in the rostral part of the $\beta$-subnucleus (at 1440 from the caudal pole of the inferior olive). Bar equals $1 \mathrm{~mm}$.

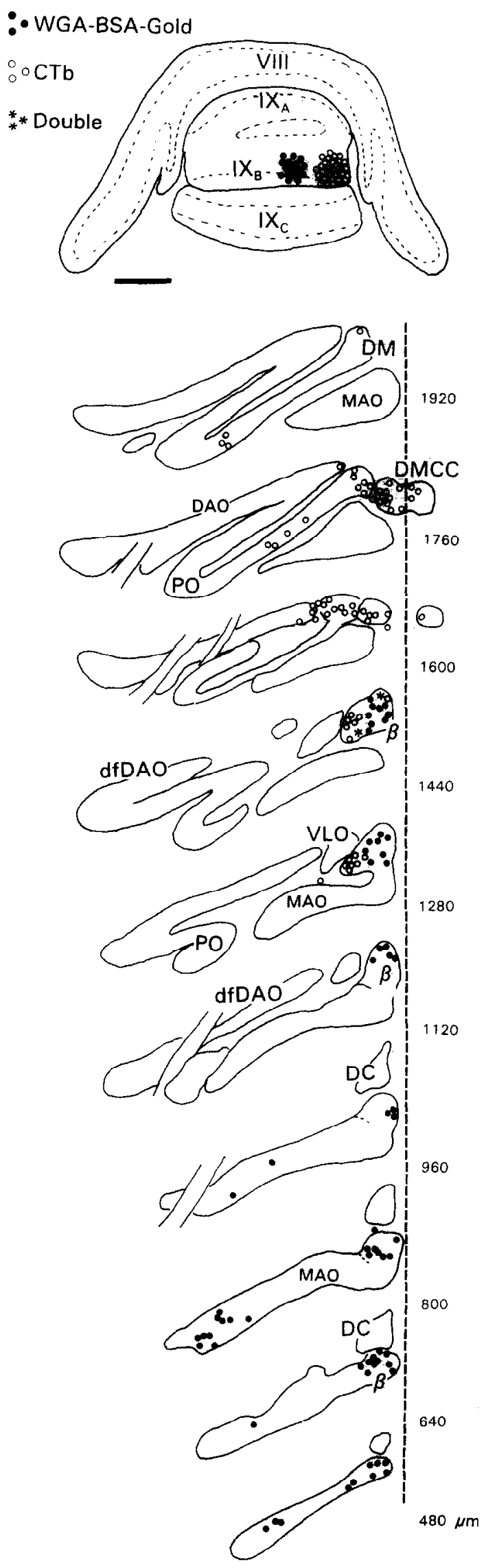


to the right side of the cord (Fig. 3A). The gold-lectin injection site was centered on the ipsilateral trochlear nucleus and surrounding area (Fig. 3B). As can be seen from Fig. 3C, showing a photomontage of the contralateral vestibular nuclei, areas containing numerous $\mathrm{CTb}$ and gold-labeled neurons can be clearly identified, even at low magnification. At higher magnification, double-labeled neurons, mostly on the left side and located within the large-celled part of the medial vestibular nucleus, can be clearly distinguished from single labeled neurons (Fig. 3D,E).

\subsubsection{Labeling in spinal cord after bilateral injections} into the lateral reticular nucleus

Various authors have shown that unilateral injections of retrograde tracers in the lateral reticular nucleus (LRN) result in bilateral labeling of neurons in the spinal cord. The question arises if, and to what extent, spinal neurons may project bilaterally to the LRN. Therefore, CTb was delivered iontophoretically (4 $\mu \mathrm{A}, 20 \mathrm{~min}$ ) into the right LRN and WGA-BSAgold $(200 \mathrm{nl})$ was pressure-injected into the left LRN. In this way, injections sites of a similar size and position could be obtained (Fig. 4A) and the resulting retrograde labeling in the spinal cord was analyzed.

It was an advantage to be able to analyze the spinal cord sections in counterstained sections with the light microscope. In this way, it was relatively easy to determine in which lamina-labeled neurons were found. Both gold-labeled as well as CTb-labeled neurons were found in various laminae at either side of the spinal cord as illustrated in Fig. 4B. Occassionally, doublelabeled neurons could also be identified (Fig. 4C,E). However, in 3 analyzed cases only 4 double-labeled neurons were encountered between $434 \mathrm{CTb}$-labeled and 454 gold-labeled neurons (10 sections/case were analyzed at various representative spinal cord levels). These data not only indicate that most spino-reticular neurons project either ipsi- or contralaterally but also that the sensitivity of both retrograde tracers are roughly comparable (i.e., a similar amount of $\mathrm{CTb}$ - and gold-labeled neurons was detected).

\subsubsection{Labeling in the inferior olive after injections in the cerebellar cortex}

The advantage of small-sized injection sites is especially clear when studying the organization of the projection from the inferior olive to the cerebellar Purkinje cells. It is well known that the climbing fibers, originating from particular olivary subdivisions, terminate on parasagittally organized strips of Purkinje cells as has been demonstrated by both anterograde and retrograde tracing techniques. However, especially with electrophysiological techniques, it was demonstrated that these strips may contain a number of functionally different subzones (Oscarsson, 1980). Therefore, it is worthwhile to study the olivocerebellar projection with increasing detail. In the experiment shown in Fig. 5, both the WGA-BSA-gold and CTb injection were made in lobule IXb of the rat cerebellum. The injection sites were both about $0.6 \mathrm{~mm}$ in diameter and appeared to touch each other. However, analysis (of every other section) indicated that only 2 doublelabeled inferior olivary cells were observed out of 74 gold-labeled and $67 \mathrm{CTb}$-labeled neurons. Furthermore, it can be observed that the labeling within the $\beta$-subnucleus reveals a rostrolateral group of neurons labeled with $\mathrm{CTb}$ and a caudomedial one containing gold/silver particles. This indicates that the climbing fiber projection from the $\beta$-subnucleus of the inferior olive to lobule IX may be subdivided into more, and potentially functionally different, zones. These observations in the rat are in line with (single) retrograde tracer studies in larger animals, such as rabbit and cat, that provide evidence that the caudal part of the $\beta$ subnucleus projects onto a midline region, whereas its rostral part projects onto a more lateral zone of the uvula-noculus (Sato and Barmack, 1985; Kanda et al., 1989). Electrophysiological evidence that neurons in the rostal and caudal parts of the $\beta$-subnucleus in the rabbit are modulated differently by vestibular stimulation has recently been published (Barmack et al., 1993).

\section{Discussion}

\subsection{Injection, uptake, and transport characteristics}

As demonstrated earlier by Menétrey and collaborators (Menétrey, 1985; Menétrey and Lee, 1985; Basbaum and Menétrey, 1987), the use of gold conjugates to trace connections in the central nervous system has many advantages over other tracers. It may be the only tracer known that is transported in retrograde direction only. It is actively taken up and transported by terminals, whereas uptake by damaged fibers appears to depend on diffusion only and consequently results in weak, if any, labeling of neurons. Passing, undamaged fibers do not appear to take up the tracer. Since it does not require a particular fixation protocol, it can be used with a variety of immunohistochemical procedures. Moreover, survival times may range from 2 days to several weeks. Silver-intensification of gold-labeled neurons results in a very characteristic labeling pattern that can be easily identified in both darkfield and lightfield microscopy.

Our gold-lectin conjugate consists of $10 \mathrm{~nm}$ gold particles conjugated to WGA that was glutaraldehydecoupled to BSA. We have also prepared and used WGA-HRP-gold (prepared according to Basbaum and Menétrey, 1987) which, after silver intensification, results in retrograde labeling with similar sensitivity as 
compared to the WGA-BSA-gold conjugate or plain WGA-HRP (although the neuronal labeling is more intense in the latter case). The main advantage of the WGA-BSA-gold over the WGA-HRP-gold and especially over WGA-apoHRP-gold appears to be its relatively low price.

Neuronal recordings with gold-lectin-filled pipettes may not be optimal since the salt content of the solution is very low $(0.005 \mathrm{M} \mathrm{NaCl})$. Nevertheless, it appears possible to use recordings of neuronal activity to verify the location of the injection site. This may be especially important when small injections are required or when two different tracers are to be injected in adjacent areas.

The use of CTb as a neuronal tracer was discussed extensively by Luppi et al. (1990). The recently available low-salt $\mathrm{CTb}$ does not require desalting and buffer-changing steps in order to be able to iontophorize the toxin. Despite the, relatively, low salt concentration, pipettes filled with $\mathrm{CTb}$ (tip diameter: about $10-14 \mu \mathrm{m}$ ) are excellent for recording neuronal activity which, as mentioned above, may aid in determining the exact location of the injection. CTb is also a good anterograde tracer (Luppi et al., 1990; Shinonaga et al., 1992) which should be taken into account when designing double-labeling experiments, e.g., it may be difficult to locate small retrogradely labeled neurons within intensely labeled fields of terminal arborizations.

Although neurons retrogradely labeled with $\mathrm{CTb}$ display a superior dendritic uptake and staining intensity compared to WGA-BSA-gold labeled neurons, we did not observe a difference in sensitivity of both tracers. When the injections were, subjectively, judged to be of a similar size and within similar areas (i.e., in the cases with bilateral injections in the LRN or within the cerebellar uvula), the number of labeled neurons containing either tracer was approximately similar.

A drawback of $\mathrm{CTb}$ may be the observation that passing, undamaged, axons may take up the tracer. This inadvertent retrograde labeling of neurons may be minimized by reducing the survival time (e.g., to $2-4$ days for tracing brain-stem connections) but should, nevertheless, be kept in mind when designing experiments.

\subsection{Double retrograde labeling}

Immunohistochemically detected and DAB-reacted $\mathrm{CTb}$ results in light to dark brown labeling of retrogradely labeled neurons. Labeling is not equally distributed but spot-like concentrations of reaction product are visible that may invade proximal and even distal dendrites. Silver/gold deposits are relatively small and intensily black, without even a shade of brown. Therefore, gold-labeling is instantly distinguishable from $\mathrm{CTb}$ labeling. Both labels can also be easily identified within a single neuron, especially when using $20 \times$ to $40 \times$ objectives. In cases of doubt, e.g., in densely CTblabeled neurons, darkfield illumination may decide whether gold/silver particles are also present in these neurons.

\subsection{Advantages over fluorescent double-labeling proce- dures}

Fluorescent tracers have been widely employed in order to study the collateralization of neurons. Kuypers et al. were the first to use substances such as Fast Blue and True Blue in combination with Nuclear Yellow or Diamino Yellow in order to study axonal branching (Bentivoglio et al., 1980a,b; Huisman et al., 1983; Keizer et al., 1983; Kuypers et al., 1977, 1980). More recently, fluorescent latex beads were introduced by Katz (Katz et al., 1984; Katz and Iarovici, 1990) and simultaneously other excellent fluorescent tracers such as Fluoro-Gold became available (Schmued and Fallon, 1986). Since these tracers either labeled different neuronal structures or possessed different spectral characteristics, multiple retrograde labeling studies became possible (Campbell and Takada, 1989; Yu et al., 1991). Also, combinations of fluorescent tracers and light microscopical tracers such as WGA-HRP (Wigston and Kennedy, 1987; Kitao et al., 1989) and gold-lectin (Berretta et al., 1991a,b; Van Bockstaele and AstonJones, 1992) have been used.

A major drawback of double or multiple labeling that involves one or more fluorescent tracers is the fact that fluorescence tends to fade, especially when excited by the proper wavelength. Although fading can be postponed when the sections are stored at $4^{\circ} \mathrm{C}$ in the dark, reexamination of the sections may not be possible interminably. The silver-intensified gold-lectin and DAB-incubated CTb immunohistochemistry do not fade for many years (at least eight), even when the sections are stored in daylight at room temperature. Another, rather important advantage of the goldlectin/CTb double-labeling technique lies in the possibility of analyzing counterstained sections. Thus, the location of labeled neurons can be easily related to surrounding brain structures, especially since changing objectives is easy and large-powered fluorescence objectives requiring oil immersion are not neccessary.

Injection sites of gold-lectin and CTb can be made at least as small as the injection sites with fluorescent latex beads and can be directed with the aid of electrophysiological recordings, which may be especially important in experiments requiring small injection sites. When designing double-labeling experiments one should be aware of the possibility that in addition to uptake by fiber terminals, inadvertent uptake by passing and/or damaged fibers (and their neuronal so- 
mata) may occur. Hence, in long survival experiments, we found evidence for uptake of CTb by passing olivocerebellar fibers (however, cf., Luppi et al., 1990). Gold-lectin may be taken up by damaged fibers, so care should be taken to use fine-tipped pipettes (diameter: $10-15 \mu \mathrm{m})$ and to inject larger quantities over a prolonged time. However, a similar, unintentional, retrograde labeling is also likely to occur using fluorescent tracers, especially since many of these substances may result in considerable necrosis at the injection site (Keizer et al., 1983).

For these reasons, we feel that the combination of a gold-lectin conjugate, such as the relatively easily prepared and cheap WGA-BSA-gold, with CTb represents a good alternative for combinations of fluorescent tracers in order to tackle problems concerning the extent of collateralization of neurons in the nervous system.

\section{Acknowledgements}

We thank Dr. J.J.L. van der Want (University of Groningen, The Netherlands) and Monica Mentink (University of Leiden, The Netherlands) for their initial help in preparing the gold-lectin conjugate. T.M.T. is supported by NWO Grant 900-550-107.

\section{References}

Barmack, N.H., Fagerson, M., Fredette, B.J., Mugnaini, E. and Shojaku, H. (1993) Activity of neurons in the beta nucleus of the inferior olive of the rabbit evoked by natural vestibular stimulation. Exp. Brain Res., 94: 203-215.

Basbaum, A.I. and Menétrey, D. (1987) Wheat germ agglutininapoHRP-gold: a new retrograde tracer for light and electronmicroscopic single- and double-label studies. J. Comp. Neurol., 261: 306-318.

Bentivoglio, M., Kuypers, H.G.J.M. and Catsman-Berrevoets, C.E. (1980a) Retrograde labeling by means of bisbenzimide and nuclear yellow (Hoechst \$769121). Measures to prevent diffusion of the tracers out of retrogradely labeled neurons. Neurosci. Lett., 18: $19-24$.

Bentivoglio, M., Kuypers, H.G.J.M., Catsman-Berrevoets, C.E., Loewe, H. and Dann, O. (1980b) Two new fluorescent tracers retrograde neuronal tracers which are transported over long distances. Neurosci. Lett., 18: 25-30.

Berretta, S., Perciavalle, V. and Poppele, R.E. (1991a) Origin of cuneate projections to the anterior and posterior lobes of the rat cerebellum. Brain Res., 556: 297-302.

Berretta, S., Perciavalle, V. and Poppele, R.E. (1991b) Origin of spinal projections to the anterior and posterior lobes of the rat cerebellum. J. Comp.Neurol., 305: 273-281.

Berretta, S., Bosco, G., Giaquinta, G., Smecca, G. and Perciavalle, V. (1993) Cerebellar influences on accessory oculomotor nuclei of the rat: a neuroanatomical, immunohistochemical, and electrophysiological study. J. Comp. Neurol., 338: 50-66.

Campbell, K.J. and Takada, M. (1989) Bilateral tectal projection of single nigrostriatal cells in the rat. Neuroscience, 33: 311-321.

Gibson, A.R., Robinson, F.R., Alam, J. and Houk, J.C. (1987)
Somatotopic alignment between climbing fiber input and nuclear output of the cat intermediate cerebellum. J. Comp. Neurol., 260: 362-377.

Huisman, A.M., Kuypers, H.G.J.M., Conde, F. and Keizer, K. (1983) Collaterals of rubrospinal neurons to the cerebellum in rat. A retrograde fluorescent double labeling study. Brain Res., 264: $181-196$

Ito, M. (1984) The Cerebellum and Neural Control. Raven Press. New York.

Kanda, K.-1., Sato, Y., Ikarashi, K. and Kawasaki, T. (1989) Zonal organization of climbing fiber projections to the uvula in the cat. J. Comp. Neurol., 279: 138-148.

Katz, L.C. and Iarovici, D.M. (1990) Green fluorescent latex microspheres: a new retrograde tracer. Neuroscience, 34: 511-520.

Katz, L.C., Burkhalter, A. and Dreyer, W.J. (1984) Fluorescent batex microspheres as a retrograde neuronal marker for in vivo and in vitro studics of visual cortex. Nature, 310; 498-500.

Keizer, K., Kuypers, H.G.J.M., Huisman, A.M. and Dann, O. (1983) Diamidino Yellow dihydrochloride (DY.2HCl); a new fluorescent retrograde tracer, which migrates only very slowly out of the cell. Exp. Brain Res., 51: 179-191.

Kitao, Y., Nakamura, Y., Kudo, M., Moriizumi, T. and Tokuno, $\mathrm{H}$. (1989) The cerebral and cerebellar connections of pretectothalamic and pretecto-olivary neurons in the anterior pretectal nucleus in the cat. Brain Res., 484: 304-313.

Kuypers, H.G.J.M., Bentivoglio, M., Catsman-Berrevoets, C.E. and Bharos, A.T. (1980) Double retrograde neuronal labeling through divergent axon collaterals, using two fluorescent tracers with the same excitation wavelenght which label different features of the cell. Exp. Brain. Res., 40: 383-392.

Kuypers, H.G.J.M., Catsman-Berrevoets, C.E. and Padt, R.E. (1977) Retrograde axonal transport of fluorescent substances in the rat's forebrain. Neurosci. Lett., 6: 127-135.

Llewellyn-Snith, I.J., Pilowsky, P. and Minson, J.B. (1992) Retrograde tracers for light and electron microscopy. In: J.P. Bolam (Ed.), Experimental Neuroanatomy. A Practical Approach, Oxford University Press, Oxford, pp. 31-59.

Luppi, P.-H., Fort, P. and Jouvet, M. (1990) Iontophoretic application of unconjugated cholera toxin $\mathrm{B}$ subunit ( $\mathrm{Clb}$ ) combined with immunohistochemistry of neurochemical substances: a method for transmitter identification of retrodradely labeled neurons. Brain Res., 534: 209-224.

Menétrey, D. (1985) Retrograde tracing of neural pathways with a protein-gold complex. I. Light microscopic detection after silver intensification. Histochemistry, 83: 391-395.

Menétrey, D. and Lee, C.L. (1985) Retrograde tracing of neural pathways with a protein gold complex. II. Electron microscopic demonstration of projections and collaterals. Histochemistry, 83: $525-530$.

Oscarsson, O. (1980) Functional organization of olivary projections to the cerebellar anterior lobe. In: J. Couville, C. de Montigny and Y. Lamarre (Eds.), The Inferior Olivary Nucleus, Anatomy and Physiology, Raven Press. New York, pp. 279-289.

Paxinos, G. and Watson, C. (1986) The Rat Brain in Stereotaxic Coordinates. Academic Press, Sydney.

Roth, J. (1983) The colloidal gold marker system for light and electron microscopic cytochemistry. In: G.R. Bullock and P. Petrusz (Eds.), Techniques in Immunocytochemistry, Academic Press, London, pp. 217-284.

Sato, Y. and Barmack, N.H. (1985) Zonal organization of olivocerebellar projections to the uvula in rabbits. Brain Res., 359: 281-191.

Schmued, L.C. and Fallon, J.H. (1986) Fluoro-Gold: a new fluorescent retrograde axonal tracer with numerous unique properties. Brain Res., 377: 147-154.

Shinonaga, Y., Takada, M. and Mizuno, N. (1992) Direct projection from the central amygdaloid nucleus to the globus pallidus and substantia nigra in the cat. Neuroscience, 51: 691-703. 
Van Bockstaele, E.J. and Aston-Jones, G. (1992) Collateralized projections from neurons in the rostral medulla to the nucleus locus coeruleus, the nucleus of the solitary tract and the periaqueductal gray. Neuroscience, 49: 653-668.

Wigston, D.J. and Kennedy, P.R. (1987) Selective reinnervation of transplanted muscles by their original motoneurons in the axolotl. J. Neurosci., 7: 1857-1965.
Yu, D.Y., Na, S., Wilson, J. and Kennedy, P.R. (1991) Redefing ral red nucleus: multiple labelling of individual neurons from spinal cord, inferior olivary nucleus and cerebellar nuclei. Neurosci. Abst., 17: 469. 